\title{
Comprehensive Analysis of the Expression, Prognostic Value and Correlations with Immune Infiltration of PBX Family Members in Colorectal Cancer
}

\author{
Yanping Hu ( $\nabla$ zlyyhuyp4176@zzu.edu.cn ) \\ Henan Cancer Hospital \\ Yihang Shen \\ Suzhou Ninth People's Hospital
}

\section{Research Article}

Keywords: PBX family, colorectal cancer, prognosis, Immune infiltration

Posted Date: September 20th, 2021

DOl: https://doi.org/10.21203/rs.3.rs-892580/v1

License: (a) (1) This work is licensed under a Creative Commons Attribution 4.0 International License.

Read Full License 


\section{Abstract}

Background: Colorectal cancer is the third commonest cancer and the second leading cause of cancer deaths globally. The Pre-B-cell leukemia transcription factor (PBX) family plays an essential biological role in the growth and development of the organism. PBX genes have been found to be implicated in the tumorigenesis of a variety of human tumors through multiple pathways, but its function in colorectal cancer is unclear.

Methods: The expression pattern, prognostic value and relationship with immune infiltration of PBX genes in patients with colorectal cancer were investigated using the Oncomine, GEPIA, Kaplan-Meier Plotter and TIMER databases. In addition, gene mutation and interaction analysis of PBX family members in colorectal cancer patients using cBioPortal and GeneMANIA databases, respectively.

Results: We revealed that a significantly lower expression level of PBX1, PBX2 and PBX3 in colorectal cancer tissues than in normal tissues, and the expression levels of PBX1 and PBX2 were significantly correlated with clinical tumor stage. Furthermore, survival analysis showed that high transcript levels of PBX4 were associated with overall survival in colon cancer patients, while low levels of PBX2 predicted improved disease-free survival in rectal cancer patients. In addition, in colon and rectal cancers, PBX proteins were notably associated with infiltration of multiple immune cells, including CD4+ T cells, CD8+ T cells, macrophages, neutrophils, B cells, and dendritic cells.

Conclusion: These findings implies that PBX1 and PBX3 are potential targets for precision therapy of colorectal cancer patients and that PBX2 and PBX4 may be new prognostic markers for colorectal cancer patients.

\section{Introduction}

Colorectal cancer, the commonly known "health killer", has become the third commonest cancer and the second leading cause of cancer deaths globally, with 1.85 million newly diagnosed cases annually[1, 2]. By 2030 , the burden of treatment for colorectal cancer is predicted to escalate by $60 \%$ globally, with in excess of 2.2 million new cases and more than 1.1 million deaths[3]. Reducing the high incidence and mortality of colorectal cancer is the most compelling need, and advanced prevention and treatment strategies are becoming imperative.

Pre-B-cell leukemia transcription factor (PBX) protein is an atypical homologous structural domain transcription factor belonging to a family of highly conserved three-amino acid-loop-extension (TALE) family of homologous structural domain proteins[4]. PBX proteins act as transcriptional cofactors for HOX proteins, forming multi-protein complexes that play important roles in the development of several organs, which include the brain, heart, and pancreas[5]. In mammals, the PBX family contains 4 members, PBX1, PBX2, PBX3 and PBX4[6]. Researchers in developmental biology have shown that PBX proteins serve an essential function in the embryonic development of the organism and that deficiency of the PBX1 or PBX3 genes causes multi-organ hypoplasia and respiratory failure in mice, eventually leading to 
death $[7,8]$. Studies in zebrafish have shown that PBX2 and PBX4 regulate brain development in a partially superfluous manner, and that defects in the PBX2 and PBX4 complexes prevent segmentation of the hindbrain in zebrafish $[9,10]$. And studies in oncology have shown that dysregulation of PBX proteins is strongly involved in the pathogenesis and progression of multiple tumors, which include gastric cancer, breast cancer, ovarian cancer and glioma[11-15]. For example, PBX1 can act as a driver to initiate ERamediated transcriptional responses in breast cancer, is involved in more than $70 \%$ of the estrogen response, and is associated with poor prognosis[14]. However, Li W, et al revealed that Pbx1 inhibited the proliferation of NSCLC cells, which suggested a complex role of Pbx1 in regulating the proliferation of NSCLC cells[16]. Besides, PBX2 co-expressed with HOXA6 can promote the proliferation, metastasis and invasion of gastric cancer cells[17]. In addition, PBX3 induces the development of epithelial mesenchymal transition, which is "silent" in normal physiological state and activated in tumorigenesis. Previous studies have shown that overexpression of PBX3 increases the proliferative capacity of gastric cancer cells and promotes cell invasion[18]. Additionally, PBX4 has been reported to be significantly reduced in leukemic cell lines and acute lymphoblastic leukemia (ALL) patients, implicating its potential role as a tumor suppressor[19]. Although aberrant expression of PBX gene family members has been noted in colorectal cancer, the association of its transcripts with prognosis and immune infiltration is not yet clear.

In the present study, we conducted bioinformatics analysis of the PBX gene family based on several public databases such as Oncomine and Gene Expression Profiling Interactive Analysis (GEPIA). We then analyzed the correlation between the expression of PBX gene family members and the prognosis of colorectal cancer patients in combination with Kaplan-Meier plotter database. In addition, we also investigated the relevance of PBX gene family members to tumor-infiltrating immune cells in the tumor microenvironment by Tumor Immune Estimation Resource (TIMER). We expect to provide a research basis for subsequent in-depth exploration of the specific molecular mechanisms underlying the involvement of the PBX gene family in the regulation of colorectal cancer progression.

\section{Materials And Methods}

\section{Oncomine database analysis}

The Oncomine database (www.oncomine.org) is a large tumor gene microarray database that combines RNA and DNA-seq data obtained from sources including published literature, GEO and TCGA[20]. The database enables the analysis of differences in gene expression, prediction of co-expressed genes, and classification based on clinical information such as tumor stage, grade, and tissue type, etc. The Oncomine database was utilized to identify the transcript levels of PBX in different cancers. The screening conditions are as follows: (1) Type of tissue: normal vs. cancer analysis; (2) Date type: mRNA and DNA; (3) Sample source: clinical specimen; (4) Critical value setting condition ( $P$ value $<0.05$; gene ranking: upper 10\%).

\section{GEPIA database analysis}


GEPIA database (http://gepia.cancer-pku.cn) is a visual oncology macrodata analysis platform based on RNA sequencing data from TCGA and GTEx to conduct cancer and normal tissue gene expression as well as interactional analysis, providing interactive and custom features including differential gene expression analysis, survival analysis, etc[21]. Initially, the GEPIA database was adopted to analyze the PBX gene family expression in colorectal cancer, and then the differential expression of PBX gene family in the pathological stage of colorectal cancer and its relationship with survival prognosis were evaluated. The screening conditions in the Expression DIY module were as follows: (1) Gene: PBX; (2) Expression DIY: Profile and Stage plot; (3) Datasets selection (cancer name): COAD and READ; 4) Matching normal data: TCGA normal data and GTEx data are matched. The screening conditions in the E Survival plots module were as follows: (1) Gene: PBX; (2) Methods: Overall survival (OS) or Disease-free survival (DFS); (3) Group cutoff: median; (4) Hazards ratio (HR): yes; (5) Datasets selection: COAD and READ. The remainder of the settings are the default options for the database.

\section{CCLE database analysis}

The CCLE database(https://sites.broadinstitute.org/ccle) is a massive publicly available tumor genome database containing a variety of high-throughput sequencing data including whole-genome sequencing data, entire-exome sequencing data, transcriptome sequencing data, and DNA methylation RRBS data[22]. The PBX gene family was analyzed by using the CCLE database to obtain the expression of the PBX gene family in colorectal cancer cell lines.

\section{HPA database analysis}

The HPA database (https://www.proteinatlas.org/) is a large-scale transcriptomic and proteomic database based on RNA sequencing analysis and immunohistochemical analysis, containing immunohistochemical and immunofluorescence expression data in normal and cancer tissues[23]. In this study, Human Protein Atlas provides immunohistochemical staining images of PBX gene family proteins in normal colorectal tissues and colorectal cancer tissues.

\section{Kaplan-Meier plotter database analysis}

The Kaplan-Meier plotter database (http://kmplot.com/analysis/) has been established on the basis of gene microarray and RNA sequencing data from public databases including TCGA, GEO and EGA to evaluate the influence of 54675 genes on survival in 21 tumors. The database was used for the investigation, identification and verification of molecular markers associated with survival by integrating gene expression information and clinical prognostic value[24]. To analyze OS and RFS in colorectal cancer patients, we separated patient samples into two groups (high versus low expression group) in accordance with the median gene expression. In this study, the Kaplan-Meier survival plot was used to analyze OS and RFS in both patient cohorts, as well as to calculate hazard ratios (hazard radio, HR) and Log rank P-values, and the number of risks were indicated below the main plot.

\section{cBioPortal database analysis}


The cBioPortal database (www.cbioportal.org) as a comprehensive online resource platform offering visible, multi-dimensional cancer genomics data, containing high-throughput data and clinical information on DNA, RNA and proteins of multiple common cancers[25]. In this study, we obtained the mutations of PBX gene family members in colorectal cancer tissues utilizing the cBioportal database. A screening condition is described below. (1) Genes: PBX; (2) Tumor type: colorectal adenocarcinoma; (3) Molecular Profiles: Mutations and Copy number alterations. The results are displayed in the interface of "OncoPrint" and "Cancer Types Summary".

\section{GeneMANIA database analysis}

The GeneMANIA database (http://www.genemania.org) is a retrieval tool for gene and protein interaction relationships that can uncover interrelationships in a collection of genes based on documented data, including co-expression, co-localization, physical interactions, protein and genetic interactions, and other properties[26]. In the present study, we utilized the GeneMANIA database to construct a correlation gene network for the PBX gene family and to predict its function.

\section{TIMER database analysis}

The TIMER database (https://cistrome.shinyapps.io/timer/) serves as a comprehensive database that systematically evaluates the immune infiltrate of multiple cancer types, providing a variety of methods to estimate the degree of immune infiltration and allowing researchers to generating dynamically online high-quality charts that comprehensively probe the immunohistological, clinical manifestations and genomic features of multiple tumors[27]. In the present study, we analyzed the association of PBX expression with the level of infiltration of immune cells in colon and rectal cancers using gene modules in the TIMER database, including B cells, CD4 + T cells, CD $8+T$ cells macrophages, neutrophils and dendritic cells for immune infiltration. In addition, we analyzed the correlation between PBX expression and genetic markers of tumor-infiltrating immune cells in colon and rectal cancers by using the correlation module in the TIMER database. Conditions for screening are as follows. (1) Cancer: COAD and READ; (2) Gene: PBX; (3) Correlation Adjusted: None or Tumor purity.

\section{Statistical analysis}

The correlation between PBX expression and colon or rectal cancer patients' prognosis was analyzed by Kaplan-Meier model and Log-rank test. $p$ value $<0.05$ was deemed to be statistically significant.

\section{Results}

\section{Transcriptional Levels of PBX in colorectal cancer patients}

So far, researchers have identified four PBX family members in mammalian cells. However, their role in colorectal cancer progression is poorly understood. To investigate whether PBX family members are implicated in the progression of colorectal cancer, we identified the expression of PBX family members in colorectal cancer tumor tissue and normal tissue using the Oncomine database, which contains 465, 449, 
443, and 258 unique datasets for PBX1, PBX2, PBX3, and PBX4. respectively. According to the Oncomine data, we found that the transcript levels of PBX1 and PBX3 were significantly reduced in colorectal cancer tissues compared to normal tissues, while the transcript levels of PBX4 were remarkably elevated (Fig. 1A). In addition, we further explored the relevant expression levels of PBX transcripts in colorectal cancer cell lines through the CCLE database, and the results are displayed in Fig. 1B.

To further examine the differential expression of PBX family members in patients with colorectal cancer, we used GEPIA dataset to compare the mRNA expression of PBX in colon and rectal cancer and their corresponding normal tissues, respectively. The results showed that PBX1, PBX2 and PBX3 were expressed at lower levels in colon and rectal cancer tumor tissues than in normal tissues, whereas PBX4 was expressed at significantly higher levels in colon and rectal cancer tumor tissues (Fig. 2). These results were generally in accordance with that of Oncomine.

\section{Correlation between the mRNA Levels of PBX and the Clinicopathological Parameters of colorectal cancer patients}

We investigated whether the expression of PBX was correlated with the pathological stages in colorectal cancer patients. The correlation between differentially expressed PBX and the pathological stages of colorectal cancer patients was evaluated using the GEPIA database. The results showed that the PBX1 and PBX2 groups were highly variable, whereas the PBX3 and PBX4 groups did not exhibit significant differences (Fig. 3). These data suggest that PBX1 and PBX2 may function essentially in the pathogenesis and progression of colorectal cancer.

To validate the transcriptional profiles of PBX family members, we analyzed the expression of PBX1, PBX2, PBX3 and PBX4 proteins in colon and rectal cancers and their corresponding normal tissues through the Human Protein Atlas database. Representative images of immunohistochemical staining showed that PBX1 and PBX3 proteins were barely detectable in colon or rectal cancer tissues, but were expressed in the corresponding normal tissues (Fig. 4). PBX2 protein was expressed in both colorectal cancer tumors and their normal tissues, but at much lower levels in tumor sections (Fig. 4). The expression level of PBX4 protein was increased in colon cancer than in normal colon tissues. Unfortunately, there was no remarkable difference in the expression of PBX4 protein between rectal cancer tissues and their corresponding normal tissues (Fig. 4).

\section{Association of PBX mRNA expression with prognostic value of colorectal cancer patients}

The present study next examined the relevance of PBX family members to clinical outcomes in colorectal cancer patients, using an online analysis tool (GEPIA) to analyze the expression profiles and clinical information of colorectal cancer patients in the TCGA cohort. As shown in Fig. 5, we present the overall 
survival (OS) and disease-free survival (DFS) curves for patients with colon and rectal cancer. The high expression level of PBX4 was negatively correlated with the OS of colon cancer patients. Besides, in rectal cancer patients, the expression of PBX2 was tightly correlated with shorter DFS, with higher expression levels resulting in shorter DFS in patients. However, PBX1 and PBX3 appeared to have no significant impact on OS and DFS in colon and rectal cancer patients.

Furthermore, to investigate the prognostic value of PBX family members in colorectal cancer, we used the Kaplan-Meier plotter database to assess the prognostic value of PBX family members. Interestingly, Kaplan-Meier curve and log rank test analysis revealed that elevated levels of PBX3 (HR $=6.27, p=0.039)$ mRNA expression were significantly associated with longer OS in colorectal cancer patients (Fig. 6).

\section{Analysis of gene mutations and interactions of PBX family members in colorectal cancer patients}

Once the prognostic value of wild-type PBX family members was determined, we next investigated the genetic changes of PBX genes in colorectal cancer. Analysis of the TCGA dataset from an online tool, cBioPortal, showed that two or more alterations were detected in different subtypes of colorectal cancer. Additionally, the total mutation rate (missense mutations, truncations, amplifications and deletions) of PBX family members was $4 \%$ in a sample of 1949 colorectal cancer patients, and the individual mutation rates were $1.7 \%, 1.2 \%, 1 \%$ and $1.5 \%$ for PBX1, PBX 2, PBX3 and PBX4, respectively (Fig. 7A and 7B).

In addition, we analyzed the correlation between the members of the PBX family through the GEPIA database. The results showed that there was a significant positive correlation between both PBX1, PBX2 and PBX3, while a significant negative correlation existed between PBX1 and PBX4 (Fig. 7C).

Subsequently, we constructed the protein-protein interaction network of PBX family members and their related genes using GeneMANIA. The results showed that PBX family members are closely implicated in sensory organ development, myeloid cell differentiation, and regulation of hematopoietic function (Fig. 7D).

Association of expression levels of PBX family members with immune cell infiltration in colon or rectal cancer

Events of immune infiltration in the tumor microenvironment (TME) function critically in the progression of tumors and influencing the patient's clinical outcome in cancer[28]. To investigate whether the expression of PBX family members was associated with the level of tumor immune infiltration, TIMER database was used to analyze the correlation between PBX family members and immune cell infiltration. The results showed that the expression of PBX1 mRNA was significantly correlated with the infiltration levels of $B$ cells, $C D 4+T$ cells and macrophages, but not with $C D 8+T$ cells (COAD), neutrophils (READ) and dendritic cells (READ) in colon and rectal cancers (Fig. 8A). Additionally, PBX2 mRNA expression showed a remarkable positively correlation with the infiltration levels of CD $4+T$ cells, $C D 8+T$ cells and macrophages in colon cancer, but not with neutrophils and dendritic cells in rectal cancer (Fig. 8B). 
Interestingly, PBX3 mRNA expression showed a significant positive correlation with the infiltration levels of all 6 immune cells in colon and rectal cancers (Fig. 8C). As for PBX4, it was observed that the expression level of its transcript dramatically correlated with the degree of infiltration of CD4 + T cells, $\mathrm{CD} 8+T$ cells and macrophages in colon cancer. However, it was only closely correlated with the infiltration levels of neutrophils in rectal cancer (Fig. 8D). Since tumor purity is an essential element influencing immuno-infiltration analysis of clinically tumor specimens, we further analyzed the correlation between PBX family members and tumor purity, and the results showed that only PBX3 mRNA expression was significantly correlated with tumor purity in colon and rectal cancers (Fig. 8).

Subsequently, we further analyzed the correlation of mRNA expression levels of PBX family members in colon and rectal cancers with markers of different types of immune cells, including typical markers of different types of immune cells such as CD8 + T cells, T cells, B cells, monocytes, tumor-associated macrophages, neutrophils, macrophages, DC cells, NK cells, etc., and functional T cell markers of different types such as Th1 cells, Th2 cells and Treg cells, using the TIMER database (Supplementary table 1-4). Adjusted for correlation by purity, we found that PBX1 and PBX3 mRNA expression levels significantly correlated with most immune marker genes of multiple immune cells and functional $T$ cells in colon and rectal cancers, whereas PBX2 and PBX4 mRNA showed correlation with only a limited set of immune cell markers (Supplementary table 1-4).

Interestingly, we found a robust correlation between PBX1 mRNA expression and the expression levels of markers in Monocyte, TAM, M2 Macrophage, Neutrophils, and Treg cells. For example, marker genes CD86 and CSF1R for Monocyte, CCL2 and CD68 for TAM, and CD163 and MS4A4A for M2 Macrophage (Supplementary table 1). In addition, except for markers of B cell, Natural killer cell and T cell exhaustion in rectal cancer, PBX3 was significantly correlated with virtually all other types of immune markers in colon or rectal cancer (Supplementary table 3 ). The above results suggest that PBX1 and PBX3 mRNA expression levels are relevant to the degree of tumor immune infiltration in colon or rectal cancer.

\section{Discussion}

Colorectal cancer is a malignant tumor threatening human health worldwide. The development of colorectal cancer is a complex multi-stage process involving multiple genes, including inactivation of anti-oncogenes, activation of oncogenes and abnormalities of various signal transduction pathways[29, 30]. In recent years, newly diagnosed patients are tending to be younger, with more than $50 \%$ of patients attributed to modifiable risk factors[31]. Prevention through screening and surveillance, as well as more timely diagnosis in younger patients, is particularly essential. Currently, the most effective means of screening for colorectal cancer include high-sensitivity stool testing or e-colonoscopy, but the efficiency and feasibility of screening are limited by the testing conditions and patient compliance[32]. Therefore, it is crucial to find new diagnostic and therapeutic targets. The investigation of colorectal cancer-related genes can facilitate the identification of key genes or new targets to improve the screening efficiency and prognosis of patients with advanced colorectal cancer. 
A homolog of the Drosophila extraadenticle gene (Exd), the PBX gene encodes four (PBX1-4) in the human genome[6]. Resembling the HOX gene, PBX encodes a transcription factor containing homeodomain, which then forms a powerful complex with the HOX1-11 protein in the context of the HOX/PBX DNA binding consensus. [6]. Previous studies have shown that multiple members of the PBX gene family are involved in a variety of tumorigenic processes[11, 12,33]. The most typical of such functions as chimeric fusion partners in variety of leukemias and lymphomas. For example, PBX1-E2A remodels the signaling network in pre-B-cell acute lymphoblastic leukemia, leading to excessive activation of the key oncogenic effector enzyme PLCY2 [34]. Studies have also been demonstrated that upregulation of PBX3 and MEIS1 in mouse models are intrinsic factors which are essential to drive leukemogenesis. Mechanistically, PBX3 protein both stabilizes MEIS1 and induces transcription of MEIS1 gene[33]. In addition, PBX genes are aberrantly expressed in solid tumors and are closely associated with patient prognosis[16]. A rigorous studies have shown that downregulation of PBX1 may increase radiosensitivity of oesophageal squamous cell carcinoma cells and xenograft cells through the PBX1/STAT3 pathway, while increased expression of PBX1 in ovarian cancer is associated with shorter survival after chemotherapy and increased resistance to platinum-based drugs by maintaining a stem cell-like phenotype[35]. PBX2 is a transcriptional activator, and high expression of PBX2 may significantly promote VP16induced apoptosis in lung cancer cells, a pathway negatively regulated by miR-1915-3p [36]. Li et al. revealed that PBX3 protein expression was significantly elevated in gastric cancer cells and was tightly intertwined with the depth of invasion, tumor grade and advanced clinical stage[37]. An additional interesting study showed that PBX3 promotes the proliferation of cervical cancer cells through the AKT signaling pathway and is expected to be a prognostic indicator[38]. In contrast, for PBX4, no study has been reported on its association with solid tumors.

Tumor-related biological databases enable efficient analysis of massive gene sequencing data. In this study, the expression of PBX gene family in colorectal cancer and its prognostic value were analyzed in depth and comprehensively using the online tool of tumor database based on relevant data from a largescale genome sequencing database. Actually, we found that PBX1-3 genes were less expressed in colorectal cancer tissues than in normal tissues, while the level of PBX4 gene was higher in colorectal cancer tissues, as analyzed by Oncomine database, GEPIA database and Human Protein Atlas database. We further analyzed the correlation between PBX gene family members and clinical stage of colorectal cancer patients, and the results suggested that PBX1 and PBX2 expression was significantly higher in advanced colon cancer patients. Overall, the above results fully suggest that PBX family members are firmly involved in the malignant progression process of colorectal cancer patients. Subsequently, we utilized GEPIA database and the Kaplan-Meier plotter database to estimate the predictive value of PBX gene family members for colorectal cancer survival. The results showed no obvious statistical difference between PBX1 gene expression and patient prognosis, and its evaluation as prognosis of colorectal cancer patients still needs further validation in clinical samples. In contrast, the expression of other PBX gene family members was significantly associated with overall survival or disease-free progression survival in colon or rectal cancer patients, and together, these findings indicated that PBX gene family members have a promising potentiality as biomarkers for the prognosis of colorectal cancer patients with 
different clinicopathological characteristics. In addition, we performed genomic variation analysis of PBX gene family members using the cBioPortal database and found that their overall mutation rate in colorectal cancer patients was $4 \%$, and mutation was dominated by mutation, amplification and profound deletion. Through GeneMANIA, an online database containing gene interactions and potential functional analyses, we evaluated the closely associated genes of PBX gene family members to explore their possible involvement in biological pathways and functions. Functional enrichment revealed that PBX gene family members are mainly associated with sensory organ development, bone marrow cell differentiation, and regulation of hematopoiesis.

Tumors are genomic diseases characterized by genomic instability, with a large accumulation of point mutations and structural alterations during tumor progression. Such genomic variants may produce tumor antigens that may be potentially identified by the immune system as non-self antigens and trigger a cytological immune response[39-41]. The immune surveillance serves a crucial function in the immune system. Upon recognition of localized cancerous lesions in organism tissues by immune surveillance, immune cells of the adaptive and innate immune systems are rapidly initiated to infiltrate the TME and assist in regulating the tumor development[42, 43]. In this study, we discovered that the PBX gene family member expression may obviously correlate with the level of immune infiltration of B cells, CD $4+T$ cells, CD8 + T cells, dendritic cells, macrophages and neutrophils by TIMER database analysis, suggesting that PBX may be involved in the immune infiltration process of colorectal cancer cells in addition to reflecting the disease prognosis of colorectal cancer patients. This study may provide detailed immunological information for colorectal cancer research to facilitate the design of new immunotherapeutic approaches.

In conclusion, the present study has carried out a comprehensive bioinformatics analysis of the PBX gene family and colorectal cancer patients, and found that the PBX gene family is aberrantly expressed in colorectal cancer tissues and is strongly correlated with the clinical stage, disease progression and survival prognosis of patients, which is expected to be a diagnostic marker and therapeutic goal for the earlier diagnosis and clinical treatment of colorectal cancer, providing a theoretical basis for the next experimental studies. However, due to the limitations of bioinformatics analysis, the function of PBX gene family in colorectal cancer and the related molecular mechanisms need to be further investigated in clinical samples and cellular and animal experiments.

\section{Abbreviations}

PBX Pre-B-cell leukemia transcription factor

TALE three-amino acid-loop-extension

GEPIA Gene Expression Profiling Interactive Analysis

TIMER tumor-infiltrating immune cells in the tumor microenvironment

COAD Colon adenocarcinoma

Page $10 / 22$ 


\section{Declarations}

\section{Author contributions}

YP Hu designed the study and wrote the manuscript. YH Shen analyzed the data.

\section{Acknowledgements}

None.

\section{Consent for publication}

N/A.

\section{CONFLICTS OF INTEREST}

The authors have declared that no conflicts of interest.

\section{FUNDING}

None.

\section{Data Availability Statement}

All relevant data are within the manuscript and its Supporting Information files.

\section{Ethical approval}

$\mathrm{N} / \mathrm{A}$.

\section{References}

1. Yu X, Zhu L, Liu J, Xie M, Chen J, Li J. Emerging Role of Immunotherapy for Colorectal Cancer with Liver Metastasis. Onco Targets Ther. 2020;13:11645-58.

2. Mattiuzzi C, Sanchis-Gomar F, Lippi G. Concise update on colorectal cancer epidemiology. Ann Transl Med. 2019;7:609.

3. Purushothaman VL, Cuomo RE, Garland CF, Mackey TK. Could age increase the strength of inverse association between ultraviolet B exposure and colorectal cancer? BMC Public Health. 2021;21:1238.

4. Capellini TD, Zappavigna V, Selleri L. Pbx homeodomain proteins: TALEnted regulators of limb patterning and outgrowth. Dev Dyn. 2011;240:1063-86.

5. Welsh IC, Hart J, Brown JM, Hansen K, Rocha Marques M, Aho RJ, Grishina I, Hurtado R, Herzlinger D, Ferretti $\mathrm{E}$, et al. Pbx loss in cranial neural crest, unlike in epithelium, results in cleft palate only and a 
broader midface. J Anat. 2018;233:222-42.

6. Longobardi E, Penkov D, Mateos D, De Florian G, Torres M, Blasi F. Biochemistry of the tale transcription factors PREP, MEIS, and PBX in vertebrates. Dev Dyn. 2014;243:59-75.

7. Riedhammer KM, Siegel C, Alhaddad B, Montoya C, Kovacs-Nagy R, Wagner M, Meitinger T, Hoefele J. Identification of a Novel Heterozygous De Novo 7-bp Frameshift Deletion in PBX1 by Whole-Exome Sequencing Causing a Multi-Organ Syndrome Including Bilateral Dysplastic Kidneys and Hypoplastic Clavicles. Front Pediatr. 2017;5:251.

8. Rhee JW, Arata A, Selleri L, Jacobs Y, Arata S, Onimaru H, Cleary ML. Pbx3 deficiency results in central hypoventilation. Am J Pathol. 2004;165:1343-50.

9. Waskiewicz AJ, Rikhof HA, Moens CB. Eliminating zebrafish pbx proteins reveals a hindbrain ground state. Dev Cell. 2002;3:723-33.

10. Vlachakis N, Choe SK, Sagerstrom CG. Meis3 synergizes with Pbx4 and Hoxb1b in promoting hindbrain fates in the zebrafish. Development. 2001;128:1299-312.

11. Xu X, Cai N, Bao Z, You Y, Ji J, Liu N. Silencing Pre-B-cell leukemia homeobox 3 decreases the proliferation of human glioma cells in vitro and in vivo. J Neurooncol. 2017;135:453-63.

12. Qiu Y, Song B, Zhao G, Deng B, Makino T, Tomita Y, Wang J, Luo W, Doki Y, Aozasa K, Morii E. Expression level of Pre B cell leukemia homeobox 2 correlates with poor prognosis of gastric adenocarcinoma and esophageal squamous cell carcinoma. Int J Oncol. 2010;36:651-63.

13. Magnani L, Patten DK, Nguyen VT, Hong SP, Steel JH, Patel N, Lombardo Y, Faronato M, Gomes AR, Woodley $L$, et al. The pioneer factor PBX1 is a novel driver of metastatic progression in ERalphapositive breast cancer. Oncotarget. 2015;6:21878-91.

14. Magnani L, Ballantyne EB, Zhang X, Lupien M. PBX1 genomic pioneer function drives ERalpha signaling underlying progression in breast cancer. PLoS Genet. 2011;7:e1002368.

15. Crijns AP, de Graeff P, Geerts D, Ten Hoor KA, Hollema H, van der Sluis T, Hofstra RM, de Bock GH, de Jong S, van der Zee AG, de Vries EG. MEIS and PBX homeobox proteins in ovarian cancer. Eur J Cancer. 2007;43:2495-505.

16. Li W, Huang K, Guo H, Cui G, Zhao S. Inhibition of non-small-cell lung cancer cell proliferation by Pbx1. Chin J Cancer Res. 2014;26:573-8.

17. Lin J, Zhu H, Hong L, Tang W, Wang J, Hu H, Wu X, Chen Y, Liu G, Yang Q, et al. Coexpression of HOXA6 and PBX2 promotes metastasis in gastric cancer. Aging. 2021;13:6606-24.

18. Lamprecht S, Kaller M, Schmidt EM, Blaj C, Schiergens TS, Engel J, Jung A, Hermeking H, Grunewald TGP, Kirchner T, Horst D. PBX3 Is Part of an EMT Regulatory Network and Indicates Poor Outcome in Colorectal Cancer. Clin Cancer Res. 2018;24:1974-86.

19. Rosales-Avina JA, Torres-Flores J, Aguilar-Lemarroy A, Gurrola-Diaz C, Hernandez-Flores G, OrtizLazareno PC, Lerma-Diaz JM, de Celis R, Gonzalez-Ramella O, Barrera-Chaires E, et al. MEIS1, PREP1, and PBX4 are differentially expressed in acute lymphoblastic leukemia: association of MEIS1 expression with higher proliferation and chemotherapy resistance. J Exp Clin Cancer Res. 2011;30:112. 
20. Rhodes DR, Yu J, Shanker K, Deshpande N, Varambally R, Ghosh D, Barrette T, Pandey A, Chinnaiyan AM. ONCOMINE: a cancer microarray database and integrated data-mining platform. Neoplasia. 2004;6:1-6.

21. Tang Z, Li C, Kang B, Gao G, Li C, Zhang Z. GEPIA: a web server for cancer and normal gene expression profiling and interactive analyses. Nucleic Acids Res. 2017;45:W98-102.

22. Barretina J, Caponigro G, Stransky N, Venkatesan K, Margolin AA, Kim S, Wilson CJ, Lehar J, Kryukov GV, Sonkin D, et al. The Cancer Cell Line Encyclopedia enables predictive modelling of anticancer drug sensitivity. Nature. 2012;483:603-7.

23. Ponten F, Jirstrom K, Uhlen M. The Human Protein Atlas-a tool for pathology. J Pathol. 2008;216:387-93.

24. Nagy A, Munkacsy G, Gyorffy B. Pancancer survival analysis of cancer hallmark genes. Sci Rep. 2021;11:6047.

25. Cerami E, Gao J, Dogrusoz U, Gross BE, Sumer SO, Aksoy BA, Jacobsen A, Byrne CJ, Heuer ML, Larsson $\mathrm{E}$, et al. The $\mathrm{cBio}$ cancer genomics portal: an open platform for exploring multidimensional cancer genomics data. Cancer Discov. 2012;2:401-4.

26. Warde-Farley D, Donaldson SL, Comes O, Zuberi K, Badrawi R, Chao P, Franz M, Grouios C, Kazi F, Lopes CT, et al. The GeneMANIA prediction server: biological network integration for gene prioritization and predicting gene function. Nucleic Acids Res. 2010;38:W214-20.

27. Li T, Fan J, Wang B, Traugh N, Chen Q, Liu JS, Li B, Liu XS. TIMER: A Web Server for Comprehensive Analysis of Tumor-Infiltrating Immune Cells. Cancer Res. 2017;77:e108-10.

28. Chew V, Toh HC, Abastado JP. Immune microenvironment in tumor progression: characteristics and challenges for therapy. J Oncol. 2012;2012:608406.

29. Hong SN. Genetic and epigenetic alterations of colorectal cancer. Intest Res. 2018;16:327-37.

30. Harris DM, Go VL. Vitamin D and colon carcinogenesis. J Nutr. 2004;134:3463S-3471S.

31. Chang VC, Cotterchio M, De P, Tinmouth J. Risk factors for early-onset colorectal cancer: a population-based case-control study in Ontario, Canada. Cancer Causes Control 2021.

32. Stracci F, Zorzi M, Grazzini G. Colorectal cancer screening: tests, strategies, and perspectives. Front Public Health. 2014;2:210.

33. Zhou J, Wu J, Li B, Liu D, Yu J, Yan X, Zheng S, Wang J, Zhang L, Zhang L, et al. PU.1 is essential for MLL leukemia partially via crosstalk with the MEIS/HOX pathway. Leukemia. 2014;28:1436-48.

34. Duque-Afonso J, Lin CH, Han K, Wei MC, Feng J, Kurzer JH, Schneidawind C, Wong SH, Bassik MC, Cleary ML. E2A-PBX1 Remodels Oncogenic Signaling Networks in B-cell Precursor Acute Lymphoid Leukemia. Cancer Res. 2016;76:6937-49.

35. Yu D, Ma Y, Feng C, Ma Z, Guo J, Chen H, He T, Guo J, Sun X, Qin Q, et al. PBX1 Increases the Radiosensitivity of Oesophageal Squamous Cancer by Targeting of STAT3. Pathol Oncol Res. 2020;26:2161-8. 
36. Xu C, Li H, Zhang L, Jia T, Duan L, Lu C. MicroRNA19153p prevents the apoptosis of lung cancer cells by downregulating DRG2 and PBX2. Mol Med Rep. 2016;13:505-12.

37. Li Y, Sun Z, Zhu Z, Zhang J, Sun X, Xu H. PBX3 is overexpressed in gastric cancer and regulates cell proliferation. Tumour Biol. 2014;35:4363-8.

38. Li H, Sun G, Liu C, Wang J, Jing R, Wang J, Zhao X, Xu X, Yang Y. PBX3 is associated with proliferation and poor prognosis in patients with cervical cancer. Onco Targets Ther. 2017;10:568594.

39. Zhang Y, Zhang Z. The history and advances in cancer immunotherapy: understanding the characteristics of tumor-infiltrating immune cells and their therapeutic implications. Cell Mol Immunol. 2020;17:807-21.

40. Stratton MR, Campbell PJ, Futreal PA. The cancer genome. Nature. 2009;458:719-24.

41. Podlaha O, Riester M, De S, Michor F. Evolution of the cancer genome. Trends Genet. 2012;28:15563.

42. Saeed M, Gao J, Shi Y, Lammers T, Yu H. Engineering Nanoparticles to Reprogram the Tumor Immune Microenvironment for Improved Cancer Immunotherapy. Theranostics. 2019;9:7981-8000.

43. Peng L, Zhang Y, Wang Z. Immune Responses against Disseminated Tumor Cells. Cancers (Basel) $2021,13$.

\section{Figures}

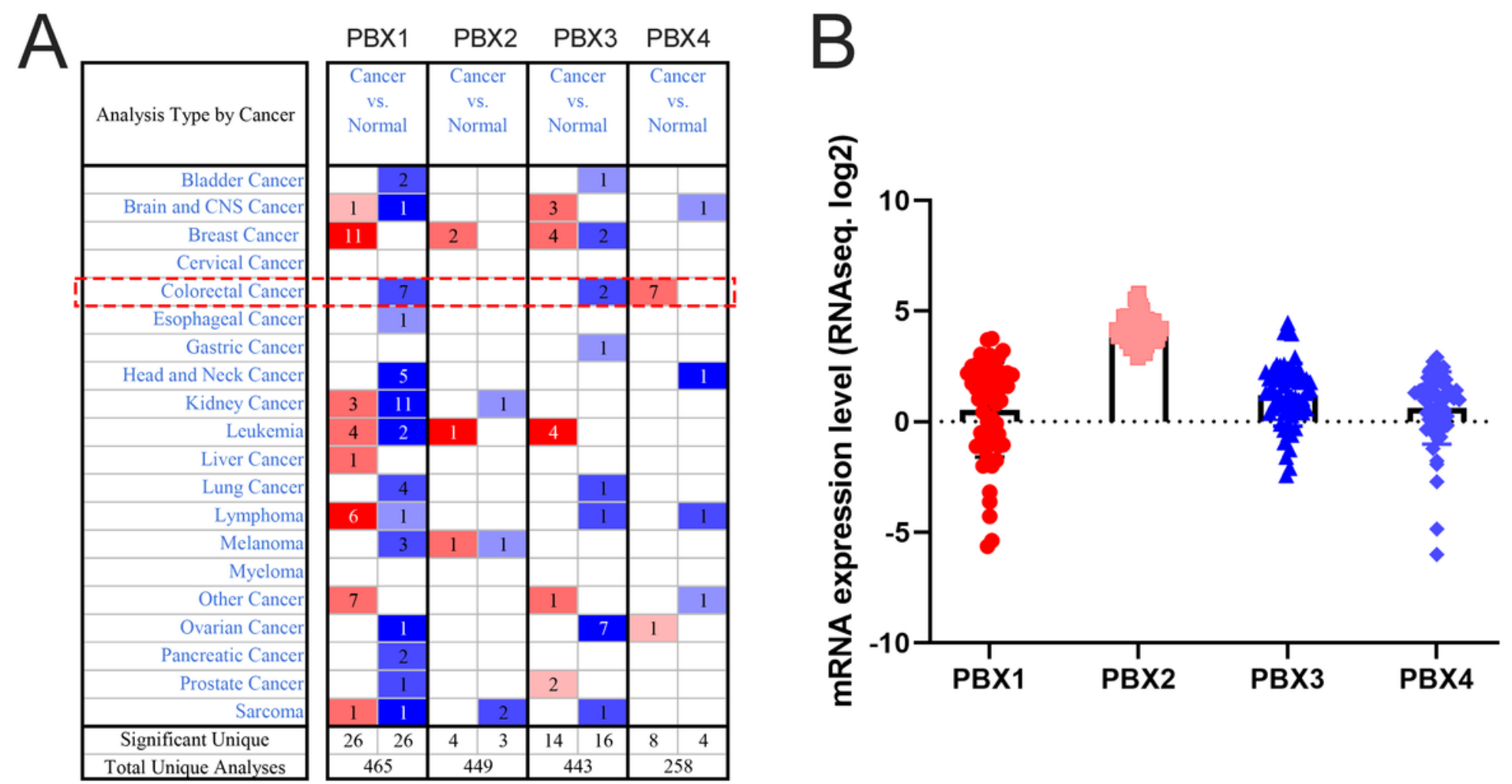


Expression levels of PBX gene family members in colorectal cancer. (A) The transcription levels of PBX gene family members in colorectal cancer (Oncomine). (B) The transcription levels of PBX gene family members in colorectal cancer cells.

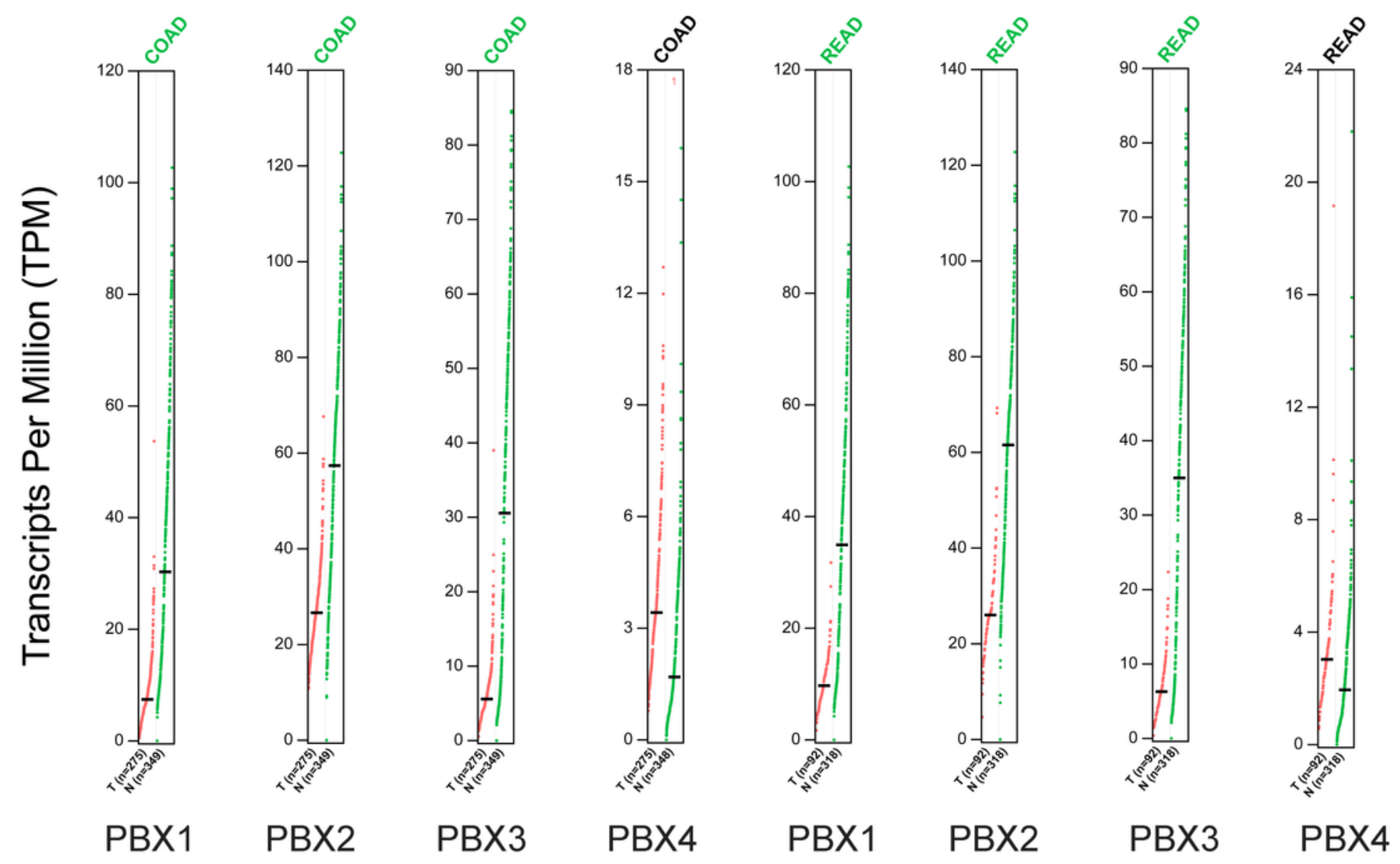

Figure 2

The Expression of PBX gene family members in colorectal cancer (GEPIA). 

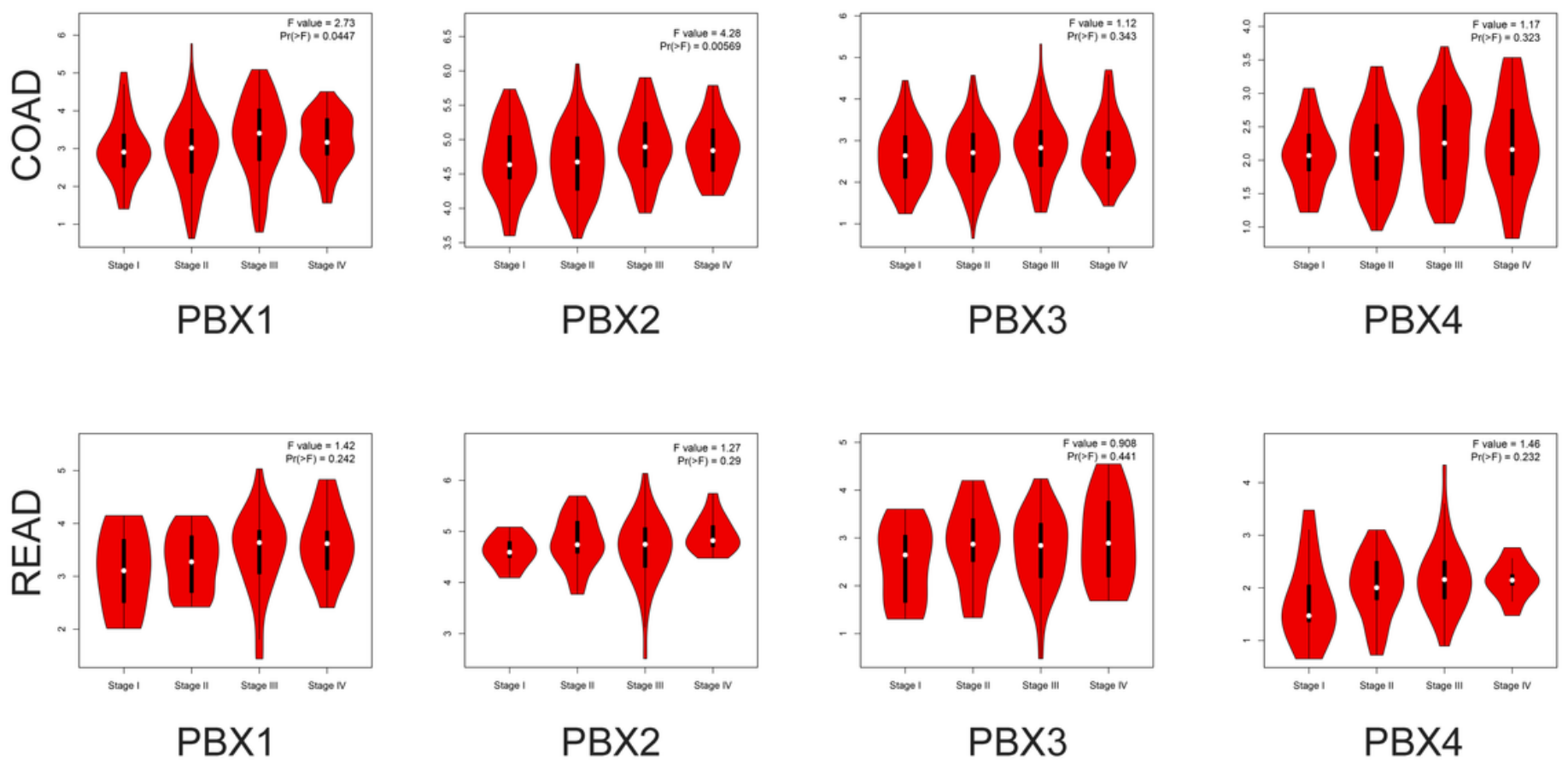

Figure 3

Correlation between the expression of PBX gene family members and tumor stage of colorectal cancer patients (GEPIA). 


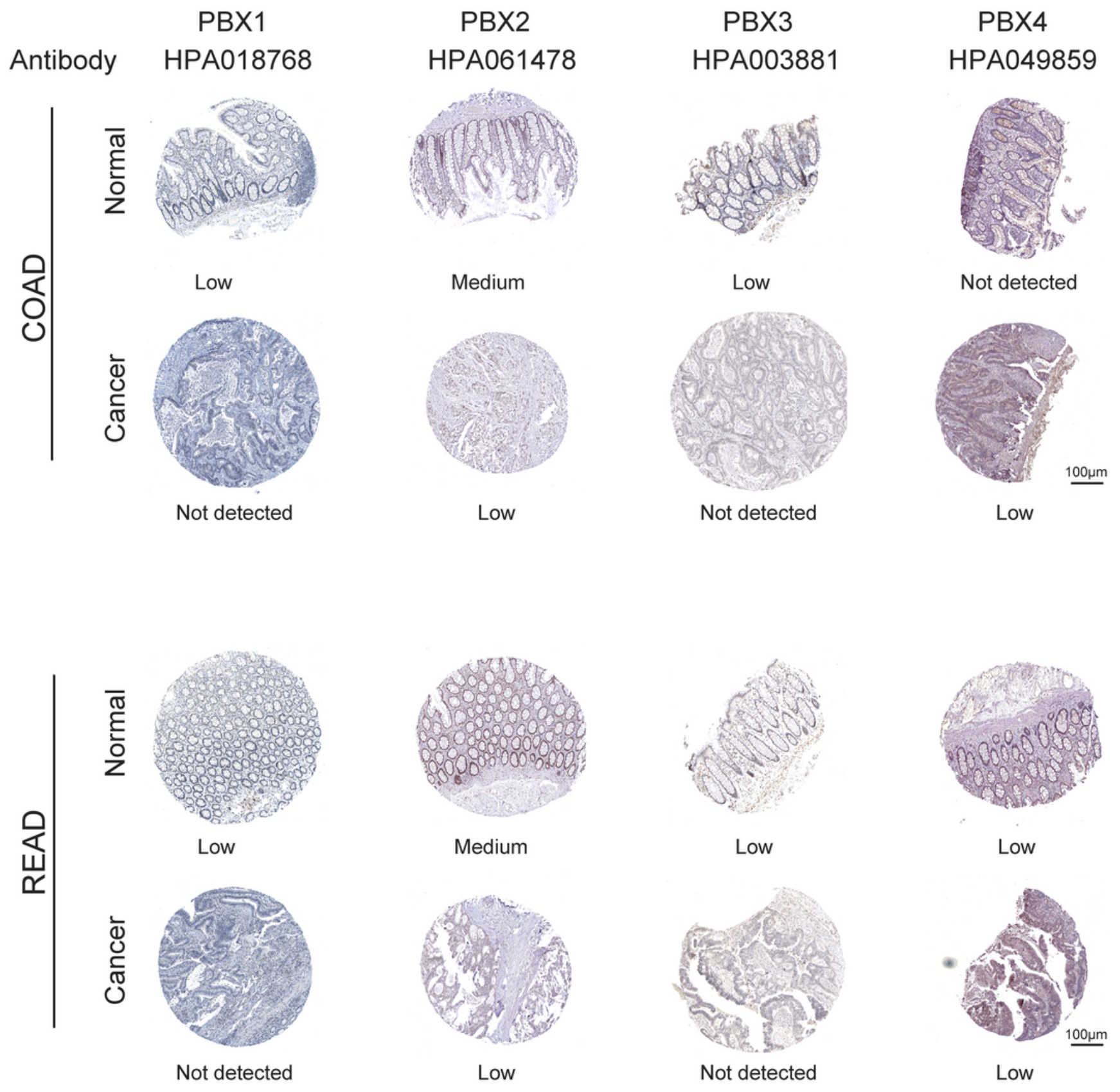

Figure 4

Representative protein expression of immunohistochemical images of PBX gene family members was examined in colorectal cancer and normal tissues (Human Protein Atlas). 
COAD
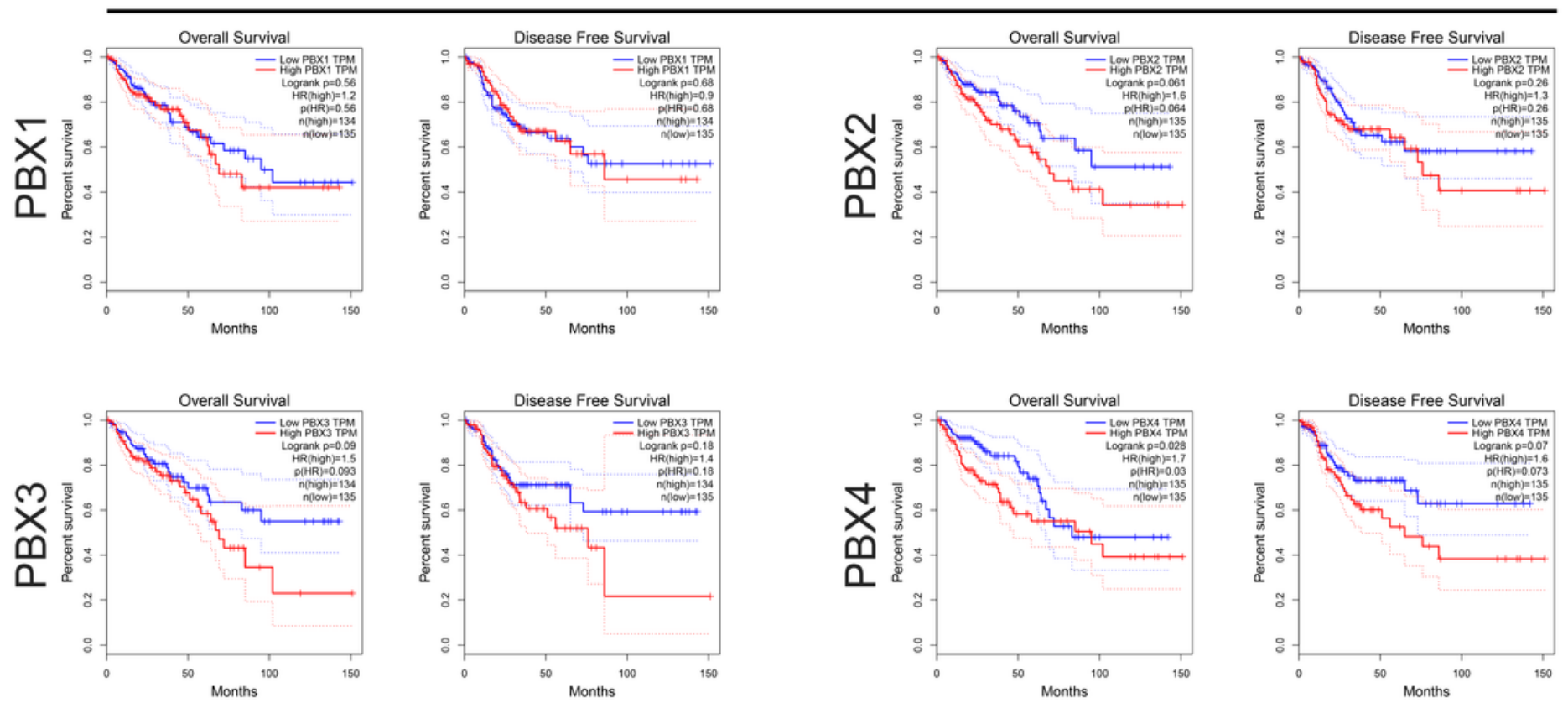

READ
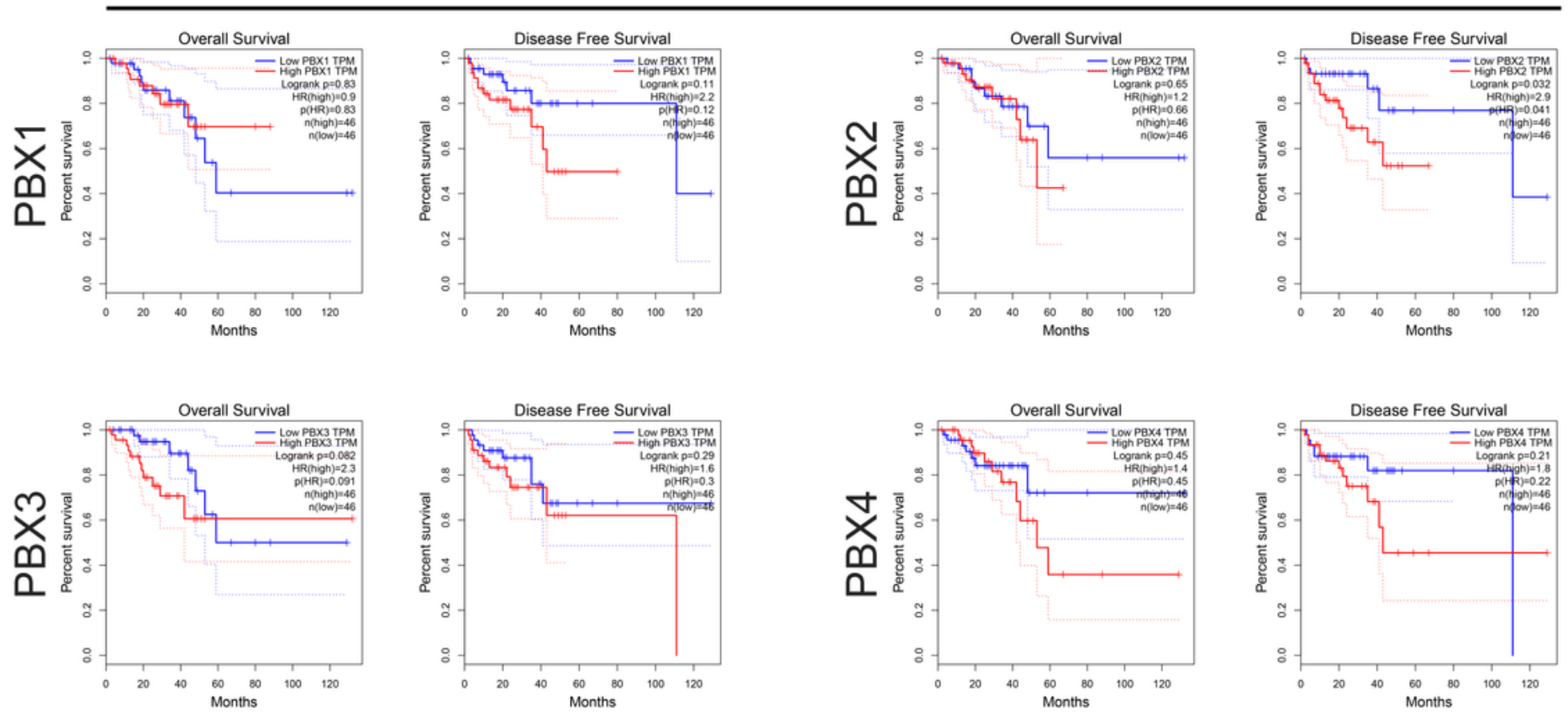

Figure 5

Analysis of the relationship between PBX gene family members and OS and DFS in colorectal cancer patients (GEPIA). 

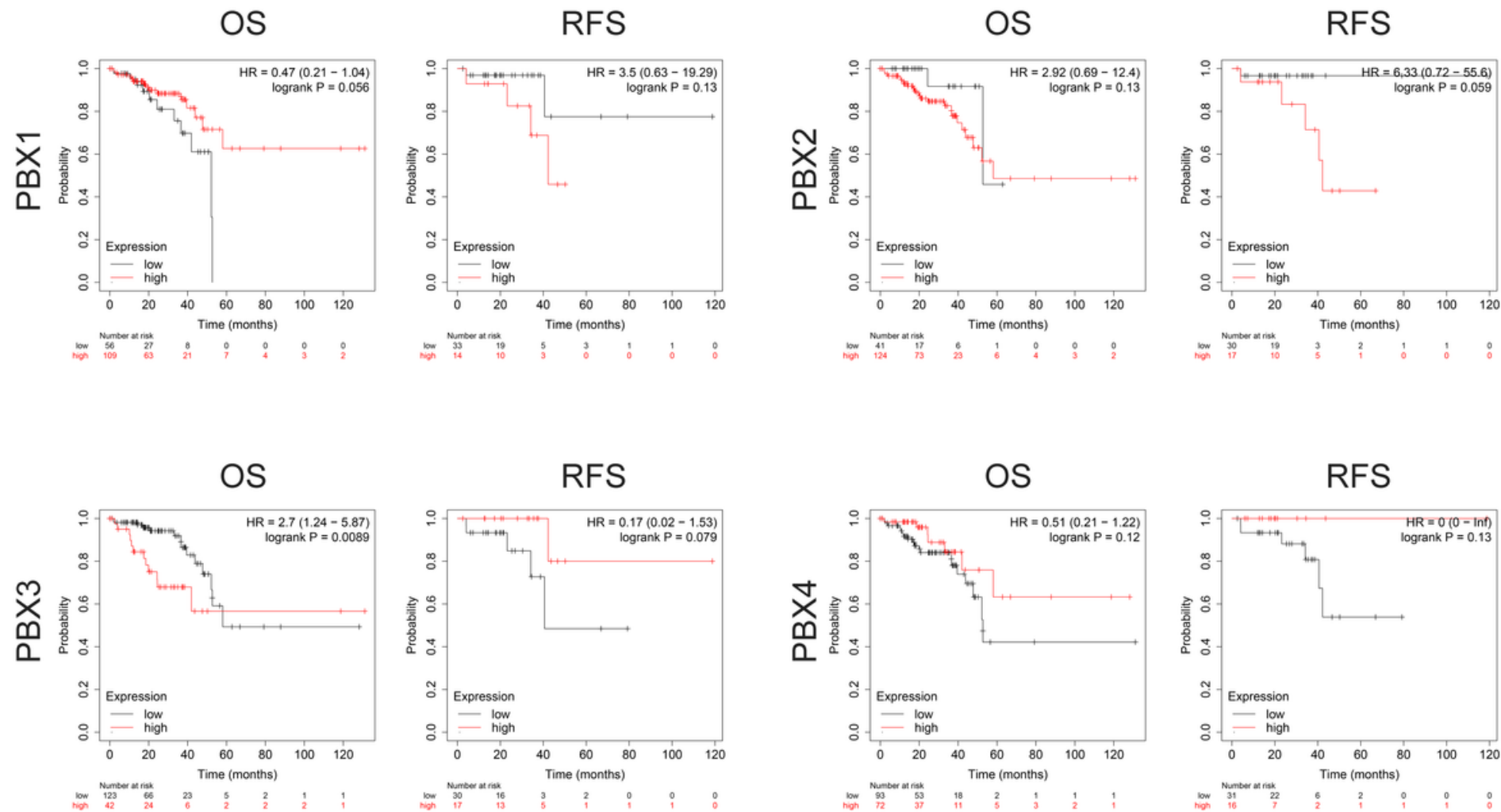

Figure 6

Prognostic value of PBX gene family members in rectal cancer (Kaplan-Meier plotter). 
A

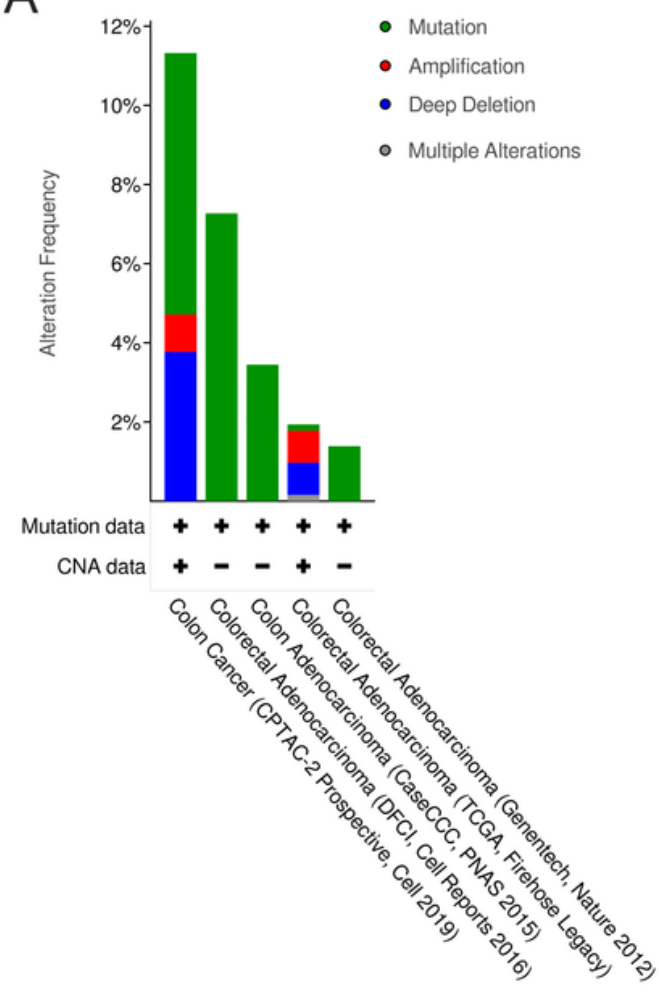

C

\begin{tabular}{|c|c|c|c|c|}
\hline & PBX1 & PBX2 & PBX3 & PBX4 \\
\hline PBX1 & 1 & 0.38 & 0.34 & -0.16 \\
\hline & 0.38 & 1 & 0.25 & -0.032 \\
\hline & 0.34 & 0.25 & 1 & -0.036 \\
\hline & -0.16 & -0.032 & -0.036 & 1 \\
\hline
\end{tabular}

B Altered in 71 (4\%) of 1949 sequenced cases/patients (total 1949)

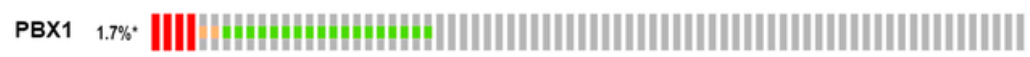

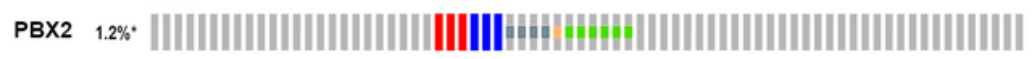
PBx3 PBx4 $1.5 \% \cdot|||||||||||||||||||||||||||||||||||||||||||||||||||||||+|||||||||||||||$
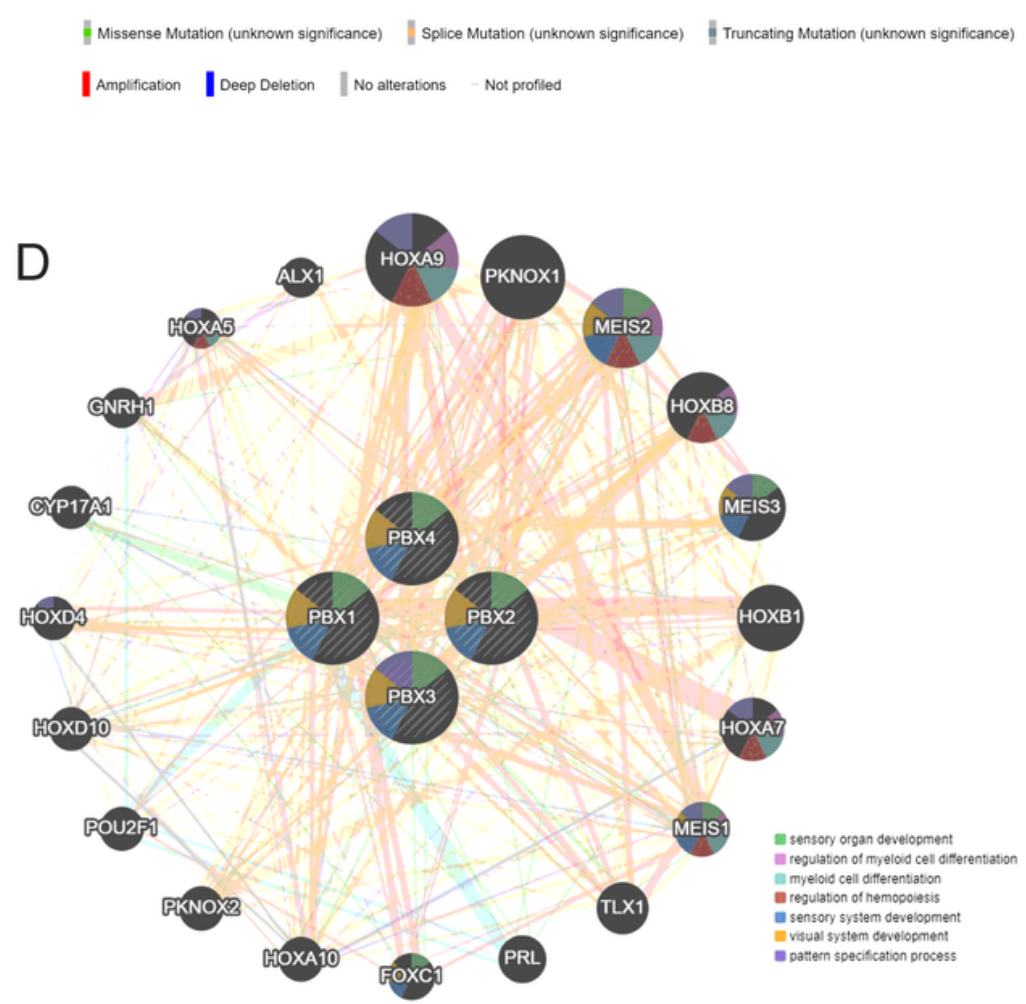

\section{Figure 7}

Mutation and expression analysis of PBX gene family members in colorectal cancer. (A) and (B) Overview of PBX gene family members expression and mutation in colorectal cancer (cBioPortal). (C) Correlation between PBX gene family members in colorectal cancer (GEPIA). (D) Protein-protein interaction network of PBX gene family members (GeneMANIA). 


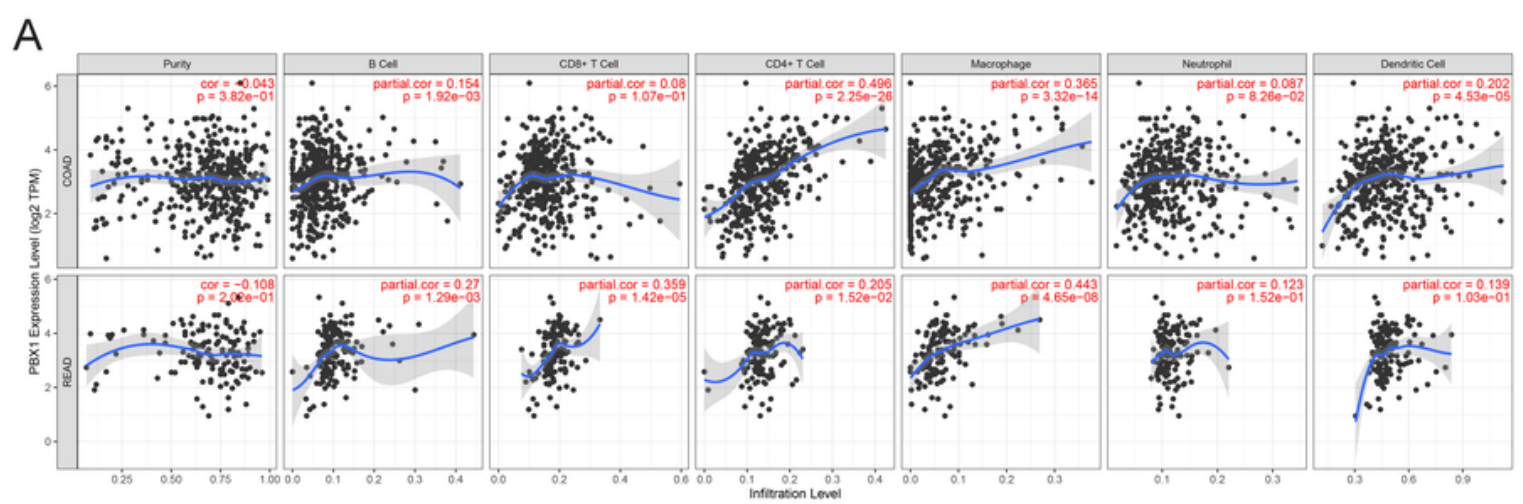

$\mathrm{B}$

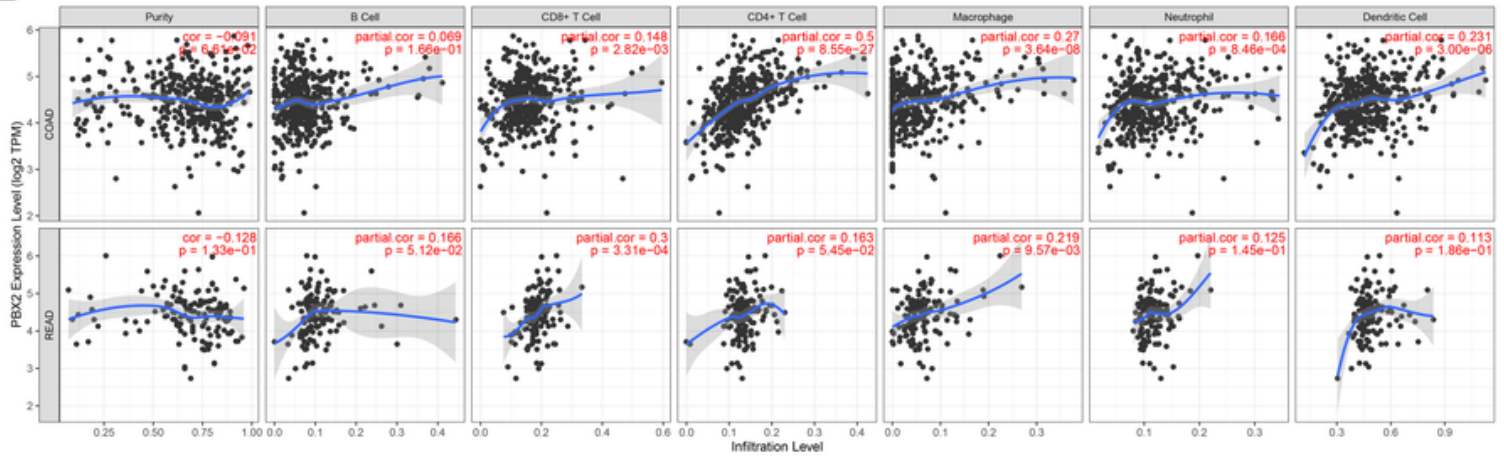

C
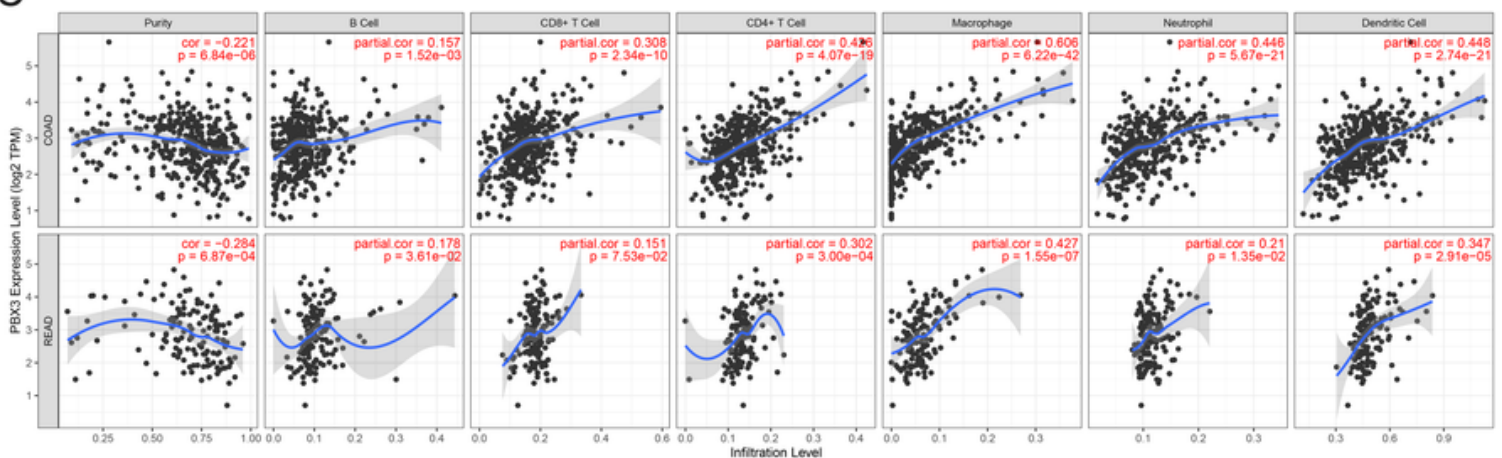

D

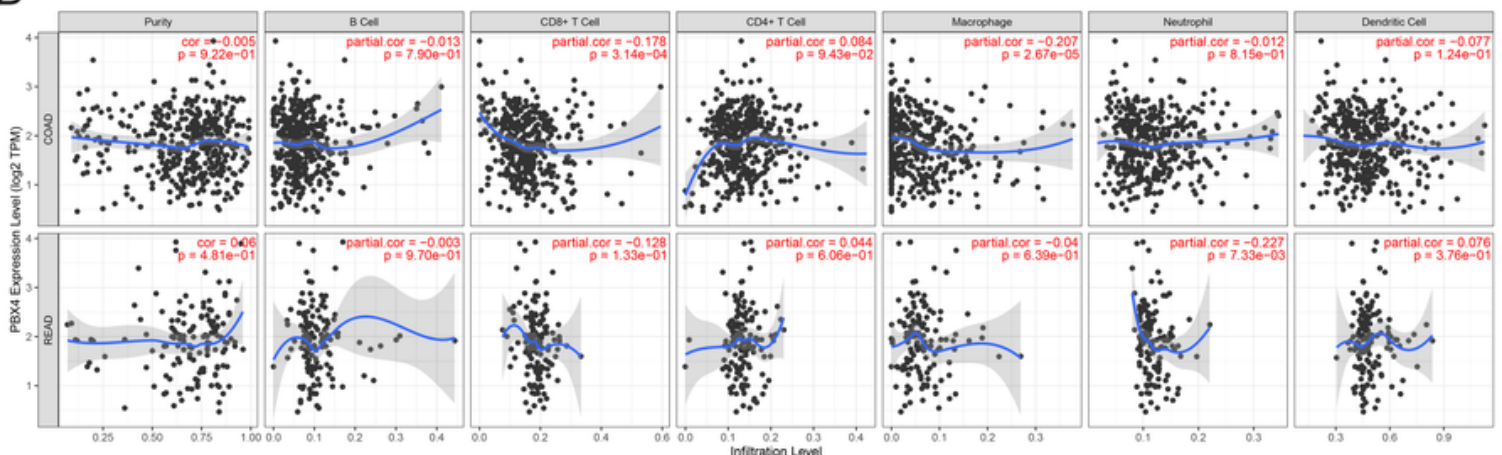

Figure 8

Association of PBX gene family members with immune cell infiltration in colorectal cancer (TIMER).

\section{Supplementary Files}

This is a list of supplementary files associated with this preprint. Click to download. 
- Supplementarytable1.xlsx

- Supplementarytable2.xlsx

- Supplementarytable3.xlsx

- Supplementarytable4.xlsx 